\title{
Space-Mapping-Based Interpolation for Engineering Optimization
}

\author{
Slawomir Koziel, Member, IEEE, John W. Bandler, Fellow, IEEE, and Kaj Madsen
}

\begin{abstract}
We consider a simple and efficient space mapping (SM)-based interpolation scheme to work in conjunction with SM optimization algorithms. The technique is useful if the fine model (the one that is supposed to be optimized) is available only on a structured grid. It allows us to estimate the response of the fine model at off-grid points and, as a result, increases the effective resolution of the design variable domain search and improves the quality of the fine model solution found by the SM optimization algorithm. The proposed method requires little computational effort. In particular, no additional fine model evaluations are necessary. Several examples that verify the accuracy and robustness of our approach are provided.
\end{abstract}

Index Terms-Engineering optimization, microwave design, offgrid interpolation, space mapping (SM), SM optimization.

\section{INTRODUCTION}

$\mathbf{S}^{\mathrm{p}}$ PACE-MAPPING (SM) technology is a novel, although already recognized, engineering optimization paradigm, consisting of a number of efficient optimization approaches [1]-[5]. The main idea behind SM is that the direct optimization of an accurate but computationally expensive high fidelity or "fine" model of interest is replaced by the iterative optimization and updating of a so-called "coarse" model (less accurate but very cheap to evaluate). Provided that the misalignment between the fine and coarse models is not significant, SM-based algorithms typically provide excellent results after only a few evaluations of the fine model.

SM was originally applied to the optimization of microwave devices [1], where fine models are often based on full-wave electromagnetic simulators, whereas coarse models are physically based circuit models. In this case the fine model evaluation can be time consuming, and saving each such evaluation counts. SM techniques have already been applied to optimization and modeling problems in a growing number of areas (see, e.g., [6]-[8]). A review and exposition of advances in SM technology is found in [5].

\footnotetext{
Manuscript received November 28, 2005; revised February 20, 2006. This work was supported in part by the Natural Sciences and Engineering Research Council of Canada under Grant OGP0007239 and Grant STGP269889 and in part by Bandler Corporation.

S. Koziel is with the Simulation Optimization Systems Research Laboratory, Department of Electrical and Computer Engineering, McMaster University, Hamilton, ON, Canada L8S 4K1 (e-mail: koziels@mcmaster.ca).

J. W. Bandler is with the Simulation Optimization Systems Research Laboratory, Department of Electrical and Computer Engineering, McMaster University, Hamilton, ON, Canada L8S 4K1, and also with Bandler Corporation, Dundas, ON, Canada L9H 5E7 (e-mail: bandler@mcmaster.ca).

K. Madsen is with Informatics and Mathematical Modelling, Technical University of Denmark, DK-2800 Lyngby, Denmark (e-mail: km@imm.dtu.dk).

Digital Object Identifier 10.1109/TMTT.2006.875298
}

Recent efforts have been focused on several areas:

1) development of new, more efficient optimization algorithms that use different SM techniques such as implicit space mapping [3], [9] and output space mapping [4];

2) development of new SM-based models [10], [11];

3) theoretical justification of space mapping and convergence theory for SM optimization algorithms [12], neuro-SM [13]-[17], and applications of SM (e.g., [18] and [19]).

In this paper, we present an SM-based interpolation technique for engineering optimization. In some situations, it happens that the model to be optimized is available on a finite grid only. This is usually due to the simulation method used to evaluate the model. For example, in the microwave area, electromagnetic simulation may be performed by solving partial differential equations by means of the method of moments (as in Sonnet em). Although, in theory, the grid can be made as fine as necessary, in practice this is not feasible because using a finer grid increases the computational cost of model evaluation as well as memory consumption. Thus, to perform optimization of the model while maintaining a reasonable grid size, we need interpolation. Normally, interpolation is based on additional fine model evaluations at different (neighboring) points. Space mapping offers a unique way of interpolating fine model responses without any additional fine model evaluations. More specifically, we use the surrogate model (that is updated after any SM iteration) to estimate the fine model response at off-grid points that are close to the current iteration point. In particular, to find the estimated fine model response at a given point, we take the fine model response at the nearest on-grid point and add a correction term which is the difference between the current surrogate model response at the point of interest and the aforementioned on-grid point. We show that the proposed interpolation method increases the resolution of the design variable domain search and improves the quality of the fine model solution found by SM optimization algorithms. On the other hand, it can be used to speed up the optimization process because an SM algorithm with our interpolation allows us to obtain the same resolution and solution quality using a much coarser grid than the SM algorithm without interpolation.

This paper is organized as follows. Section II briefly reviews the formulation of the SM concept and typical optimization algorithms. Section III introduces the proposed SM-based interpolation scheme. Section IV contains verification examples. In Section V, we present a mathematical motivation of our interpolation scheme. Section VI demonstrates that our interpolation scheme can be efficiently used to speed up the optimization process. Section VII examines robustness. A discussion and conclusions follow in Section VIII. 


\section{BASICS OF SM OPTIMIZATION}

Let us state the optimization problem as follows. Let $\boldsymbol{R}_{f}$ : $X_{f} \rightarrow R^{m}$ denote the response vector of a fine model of the device of interest, where $X_{f} \subseteq R^{n}$. Our goal is to solve the problem

$$
\boldsymbol{x}_{f}^{*}=\arg \min _{\boldsymbol{x} \in X_{f}} U\left(\boldsymbol{R}_{\boldsymbol{f}}(\boldsymbol{x})\right)
$$

where $U: R^{m} \rightarrow R$ is a given objective function. We assume throughout the paper that $X_{f}$ is a closed subset of $R^{n}$. We shall denote by $X_{f}^{*}$ the set of all $\boldsymbol{x} \in X_{f}$ satisfying (1) and call it the set of fine model minimizers.

We consider the fine model to be expensive to compute and solving (1) by direct optimization to be impractical. Instead, we use surrogate models, i.e., models that are not as accurate as the fine model but are computationally cheap, and hence suitable for iterative optimization. We consider a general optimization algorithm that generates a sequence of points $\boldsymbol{x}^{(i)} \in X_{f}, i=$ $1,2, \ldots$, and a family of surrogate models $\boldsymbol{R}_{s}^{(i)}: X_{s}^{(i)} \rightarrow R^{m}$, $i=0,1, \ldots$, so that

$$
\boldsymbol{x}^{(i+1)}=\arg \min _{\boldsymbol{x} \in X_{f} \cap X_{s}^{(i)}} U\left(\boldsymbol{R}_{s}^{(i)}(\boldsymbol{x})\right)
$$

and $\boldsymbol{R}_{s}^{(i+1)}$ is constructed using suitable matching conditions with the fine model at $\boldsymbol{x}^{(i+1)}$ (and, perhaps, some of the $\boldsymbol{x}^{(k)}$, $k=1, \ldots, i)$. We assume here that $X_{f} \cap X_{s}^{(i)} \neq \varnothing$ for $i=$ $0,1,2, \ldots$. If the solution to (2) is non-unique we may impose regularization. We may match responses, i.e.,

$$
\boldsymbol{R}_{s}^{(i)}\left(\boldsymbol{x}^{(i)}\right)=\boldsymbol{R}_{f}\left(\boldsymbol{x}^{(i)}\right)
$$

and/or match first-order derivatives

$$
\boldsymbol{J}_{\boldsymbol{R}_{s}^{(i)}}\left(\boldsymbol{x}^{(i)}\right)=\boldsymbol{J}_{\boldsymbol{R}_{\boldsymbol{f}}}\left(\boldsymbol{x}^{(i)}\right)
$$

where $\boldsymbol{J}_{\boldsymbol{R}_{\mathrm{s}}^{(i)}}$ and $\boldsymbol{J}_{\boldsymbol{R}_{f}}$ denote Jacobians of the surrogate and fine models, respectively. More precisely, we aim to define models so that conditions such as (3) and (4) are satisfied.

The family of surrogate models $\left\{\boldsymbol{R}_{s}^{(i)}\right\}$ can be implemented in various ways. SM assumes the existence of a so-called coarse model that describes the same object as the fine model: less accurate but much faster to evaluate. Let $\boldsymbol{R}_{c}: X_{c} \rightarrow R^{m}$ denote the response vectors of the coarse model, where $X_{c} \subseteq R^{n}$. By $X_{c}^{*}$, we denote the set of coarse model minimizers (i.e., the set of optimal solutions of the coarse model)

$$
X_{c}^{*}=\left\{\boldsymbol{x} \in X_{c}: \boldsymbol{x}=\arg \min _{\boldsymbol{z} \in X_{c}} U\left(\boldsymbol{R}_{c}(\boldsymbol{z})\right)\right\} .
$$

In the SM framework, the family of surrogate models is constructed from the coarse model in such a way that each $\boldsymbol{R}_{s}^{(i)}$ is a suitable distortion of $\boldsymbol{R}_{c}$, such that given matching conditions are satisfied. A variety of SM-based surrogate models have been used and described in the literature [1]-[5]. In this paper, we utilize a general SM surrogate model that incorporates both input
[2] and output [4] SM. At iteration $i, i=1,2, \ldots$, the surrogate model $\boldsymbol{R}_{s}^{(i)}$ is defined as (at iteration 0, we have $\boldsymbol{R}_{s}^{(0)}=\boldsymbol{R}_{c}$ )

$R_{s}^{(i)}(x)=A^{(i)} \cdot R_{c}\left(B^{(i)} \cdot x+c^{(i)}\right)+d^{(i)}+E^{(i)} \cdot\left(x-x^{(i)}\right)$

where

$$
\begin{aligned}
\left(\boldsymbol{A}^{(i)}, \boldsymbol{B}^{(i)}, \boldsymbol{c}^{(i)}\right)= & \arg \min _{(\boldsymbol{A}, \boldsymbol{B}, \boldsymbol{c})} \varepsilon^{(i)}(\boldsymbol{A}, \boldsymbol{B}, \boldsymbol{c}) \\
\boldsymbol{d}^{(i)}= & \boldsymbol{R}_{f}\left(\boldsymbol{x}^{(i)}\right) \\
& -\boldsymbol{A}^{(i)} \cdot \boldsymbol{R}_{c}\left(\boldsymbol{B}^{(i)} \cdot \boldsymbol{x}^{(i)}+\boldsymbol{c}^{(i)}\right) \\
\boldsymbol{E}^{(i)}= & \boldsymbol{J}_{\boldsymbol{R}_{f}}\left(\boldsymbol{x}^{(i)}\right) \\
& -\boldsymbol{A}^{(i)} \cdot \boldsymbol{J}_{\boldsymbol{R}_{c}}\left(\boldsymbol{B}^{(i)} \cdot \boldsymbol{x}^{(i)}+\boldsymbol{c}^{(i)}\right) \cdot \boldsymbol{B}^{(i)} .
\end{aligned}
$$

Matrices $\boldsymbol{A}^{(i)}=\operatorname{diag}\left\{a_{1}^{(i)}, \ldots, a_{m}^{(i)}\right\}, \boldsymbol{B}^{(i)} \in M_{n \times n}$ and $\boldsymbol{c}^{(i)} \in M_{n \times 1}$ are obtained using a process called parameter extraction (PE), as defined in (7). Matrices $\boldsymbol{d}^{(i)} \in M_{m \times 1}$ and $E^{(i)} \in M_{m \times n}$ are calculated using (8) and (9) after having determined $\boldsymbol{A}^{(i)}, \boldsymbol{B}^{(i)}$, and $\boldsymbol{c}^{(i)}$. Matching measure $\varepsilon^{(i)}$ determines the surrogate model as much as (6) does. We can consider different matching measures that aim to match the fine and surrogate model responses and/or their first-order derivatives. A general form of the matching measure is

$$
\begin{aligned}
\varepsilon^{(i)}(\boldsymbol{A}, \boldsymbol{B}, \boldsymbol{c}) & \\
= & \sum_{k=0}^{i} w_{k}\left\|\boldsymbol{R}_{f}\left(\boldsymbol{x}^{(k)}\right)-\boldsymbol{A} \cdot \boldsymbol{R}_{c}\left(\boldsymbol{B} \cdot \boldsymbol{x}^{(k)}+\boldsymbol{c}\right)\right\| \\
& +\sum_{k=0}^{i} v_{k}\left\|\boldsymbol{J}_{\boldsymbol{R}_{f}}\left(\boldsymbol{x}^{(k)}\right)-\boldsymbol{A} \cdot \boldsymbol{J}_{\boldsymbol{R}_{c}}\left(B \cdot \boldsymbol{x}^{(k)}+\boldsymbol{c}\right) \cdot \boldsymbol{B}\right\| .
\end{aligned}
$$

We assume that coefficients $w_{k}$ and $v_{k}$ are either zero or one (although more general situations are conceivable). Setting $w_{k}=1, k=0, \ldots, i$, and $v_{k}=0, k=0, \ldots, i-1, v_{i}=1$ means that the surrogate tries to match the fine model response at all previous points $\boldsymbol{x}^{(k)}$ (including the current point) as well as the Jacobian at the current point.

The general SM surrogate model can be specified by enabling/disabling particular components of the space mapping as well as by choosing proper values of weight coefficients $w_{k}$ and $v_{k}$. Any of the model components $\boldsymbol{A}, \boldsymbol{B}, \boldsymbol{c}, \boldsymbol{d}$, and $\boldsymbol{E}$ can be enabled or disabled as shown in Table I. A number of responses and Jacobians used in parameter extraction can be determined as shown in Table II. We shall use the following naming convention for the surrogate models: the presence of any of the letters $A, B, c, d, E$ is equivalent to enabling of the corresponding model component. The first (second) subscript denotes number of responses (Jacobians) used in parameter extraction (subscript "all" is allowed as shown in Table II). For example, surrogate model $\boldsymbol{B} \boldsymbol{c} \boldsymbol{d} \boldsymbol{E}_{\text {all. } 0}$ corresponds to the model that uses nontrivial components $\boldsymbol{B}, \boldsymbol{c}, \boldsymbol{d}$, and $\boldsymbol{E}$, tries to match 
TABLE I

NAming Convention of the SurRogate Models USEd IN THE SM OPTIMIZATION ALGORITHM (SURROGATE MODEL)

\begin{tabular}{ccc}
\hline \hline & \multicolumn{2}{c}{ Effect on the surrogate model } \\
\cline { 2 - 3 } Symbol & Enabled & Disabled \\
\hline $\boldsymbol{A}$ & $\boldsymbol{A}^{(i)}$ included in PE & $\boldsymbol{A}^{(i)}=\operatorname{diag}_{1}\{1 \ldots 1\}$ \\
$\boldsymbol{B}$ & $\boldsymbol{B}^{(i)}$ included in PE & $\boldsymbol{B}^{(i)}=\mathbf{I}_{n}$ \\
$\boldsymbol{c}$ & $\boldsymbol{c}^{(i)}$ included in PE & $\boldsymbol{c}^{(i)}=\mathbf{0}_{n \times 1}$ \\
$\boldsymbol{d}$ & $\boldsymbol{d}^{(i)}$ calculated according to (8) & $\boldsymbol{d}^{(i)}=\mathbf{0}_{m \times \mathbf{1}}$ \\
$\boldsymbol{E}$ & $\boldsymbol{E}^{(i)}$ calculated according to (9) & $\boldsymbol{E}^{(i)}=\mathbf{0}_{m \times n}$ \\
\hline \hline
\end{tabular}

$\mathbf{I}_{n}$ denotes identity matrix of size $n ; \mathbf{0}_{k \times l}$ denotes $k \times l$ zero matrix

TABLE II

Naming Convention of the SurRogate Models USEd IN THE SM OPTIMIZATION ALGORITHM (PE)

\begin{tabular}{ccc}
\hline \hline \multirow{2}{*}{ Symbol } & \multicolumn{2}{c}{ Effect on the surrogate model } \\
\cline { 2 - 3 } & Number of responses in PE & Number of Jacobians in PE \\
\hline 0 & $w_{k}=0, k=0,1, \ldots, i$, & $v_{k}=0, k=0,1, \ldots, i$ \\
$p$ & $w_{k}=0, k=0,1, \ldots, i-p$, & $v_{k}=0, k=0,1, \ldots, i-p$, \\
& $w_{k}=1, k=i-p+1, \ldots, i$ & $v_{k}=1, k=i-p+1, \ldots, i$ \\
all & $w_{k}=1, k=0,1, \ldots, i$, & $v_{k}=1, k=0,1, \ldots, i$ \\
\hline \hline
\end{tabular}

fine model responses from all previous iterations, and uses no Jacobian data in the parameter extraction process.

In order to improve the convergence of the optimization algorithm we enhance it by an (optional) trust region (TR) method [20] so that the original problem (2) is replaced by

$$
\boldsymbol{x}^{(i+1)}=\arg \min _{\boldsymbol{x} \in X_{f} \cap X_{s}^{(i)},\left\|\boldsymbol{x}-\boldsymbol{x}^{(i)}\right\| \leq \delta^{(i)}} U\left(\boldsymbol{R}_{\boldsymbol{s}}^{(i)}(\boldsymbol{x})\right)
$$

where $\delta^{(i)}$ denotes the TR radius at iteration $i$. We use the traditional updating rules for the TR radius since we found them to be efficient in our experiments. Let $f^{(i)}=U\left(\boldsymbol{R}_{f}\left(\boldsymbol{x}^{(i)}\right)\right)$ and $L^{(i)}=U\left(\boldsymbol{R}_{s}^{(i-1)}\left(\boldsymbol{x}^{(i)}\right)\right)$ denote the objective function value for the fine model and the surrogate, respectively, at $\boldsymbol{x}^{(i)}$ (iteration point $i$ ). The next point $\boldsymbol{x}^{(i+1)}$ is found as a solution to (11). Having $f^{(i)}, L^{(i)}, f^{(i+1)}$, and $L^{(i+1)}$, we can calculate the so-called gain ratio

$$
\rho^{(i)}=\left(f^{(i)}-f^{(i+1)}\right) /\left(L^{(i)}-L^{(i+1)}\right)
$$

The TR radius for the next iteration $\delta^{(i+1)}$ is determined in the following way:

$$
\begin{aligned}
& \text { if } \rho^{(i)}<\rho_{\text {decr }} \\
& \qquad \delta^{(i+1)}=\delta^{(i)} / m_{\text {decr }} \\
& \text { elseif } \rho^{(i)}>\rho_{\text {incr }} \\
& \quad \delta^{(i+1)}=\delta^{(i)} \cdot m_{\text {incr }} \\
& \text { else } \\
& \qquad \delta^{(i+1)}=\delta^{(i)} .
\end{aligned}
$$

In our experiments we use $\rho_{\text {decr }}=0.25, \rho_{\text {incr }}=0.75$, $m_{\text {decr }}=2$, and $m_{\text {incr }}=3$. Initial value $\delta^{(0)}$ of the TR radius is typically equal to $0.1\left\|x^{(i)}\right\|$.
The SM optimization algorithm ${ }^{1}$ can be summarized as follows:

$$
\begin{array}{ll}
\text { Step 0 } & \text { Set } i=0 ; \\
\text { Step } 1 & \text { Given } \boldsymbol{x}^{(i)}, \boldsymbol{R}_{s}^{(i)}, \text { and } \delta^{(i)} \\
& \text { obtain } \boldsymbol{x}^{(i+1)} \text { using (11) } \\
\text { Step 2 } & \text { Update } \delta^{(i)} \text { according to (12) and (13); } \\
\text { Step } 3 & \text { If } \left.\boldsymbol{x}^{(i+1)} \text { is accepted (i.e., } \rho^{(i)} \geq \rho_{\text {decr }}\right) \\
& \text { set } i=i+1, \delta^{(i)}=\delta^{(i-1)}
\end{array}
$$

and determine the new surrogate model

$\boldsymbol{R}_{s}^{(i)}$ as in (6)-(10);

Step 4 If the termination condition is not satisfied go to Step 1; else terminate the algorithm.

\section{SM-BASED INTERPOLATION}

From now on, we shall deal with the situation when the fine model can be evaluated on a discrete subset of $R^{n}$ only so that in order to conduct optimization outside this subset, we need to perform some kind of interpolation. In this section, we give the details of a simple SM-based interpolation scheme suitable to work with the optimization algorithms described in Section II.

Suppose that the fine model $R_{f}$ is only available on a subset $\bar{X}_{f}$ of its domain $X_{f}$. We will assume for the rest of this paper that $\bar{X}_{f}$ is a uniform grid with step $d_{i}$ in direction $i$ (although this assumption is not critical). Let $s: X_{f} \rightarrow \bar{X}_{f}$ (snapping function) be defined in such a way that for any $\boldsymbol{x} \in X_{f}, s(\boldsymbol{x})$ is a point $\boldsymbol{x}$ snapped to the nearest grid point (with respect to a given norm), i.e.,

$$
\begin{aligned}
& s(\boldsymbol{x})=\left\{\overline{\boldsymbol{x}} \in \bar{X}_{f}:\|\boldsymbol{x}-\overline{\boldsymbol{x}}\|=\min _{\boldsymbol{z} \in \bar{X}_{f}}\|\boldsymbol{z}-\overline{\boldsymbol{x}}\|\right.
\end{aligned}
$$

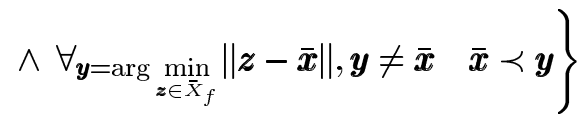

where relation $\prec$ is a lexicographic order with respect to vector components. Note that $s(\boldsymbol{x})=\boldsymbol{x}$ for any $\boldsymbol{x} \in \bar{X}_{f}$.

We would like to estimate the value of $\boldsymbol{R}_{f}$ at any point of $X_{f}$ using available data (recall that we assumed that $\boldsymbol{R}_{f}$ is only available on $\bar{X}_{f} \subset X_{f}$ ) and the current surrogate model $\boldsymbol{R}_{s}^{(i)}$ available during the optimization process. Let $\left\{\boldsymbol{x}^{(i)}\right\}$ be the sequence created during the SM-based optimization procedure. We define an interpolated fine model response $\overline{\boldsymbol{R}}_{f}$ at point $\boldsymbol{x}^{(i+1)}$ as

$$
\begin{aligned}
\overline{\boldsymbol{R}}_{f}\left(\boldsymbol{x}^{(i+1)}\right)= & \boldsymbol{R}_{f}\left(s\left(\boldsymbol{x}^{(i+1)}\right)\right) \\
& +\boldsymbol{R}_{s}^{(i)}\left(\boldsymbol{x}^{(i+1)}\right)-\boldsymbol{R}_{s}^{(i)}\left(s\left(\boldsymbol{x}^{(i+1)}\right)\right) .
\end{aligned}
$$

${ }^{1}$ The termination condition we use in this paper is as follows. The optimization algorithm is terminated if one of the conditions is satisfied:

(i) $\left\|\boldsymbol{x}^{(i)}-\boldsymbol{x}^{(i-1)}\right\| \leq \operatorname{Tol} X$ and $\left\|\boldsymbol{R}_{f}^{(i)}-\boldsymbol{R}_{f}^{(i-1)}\right\| \leq T$ olFun (convergence of the algorithm; TolX and TolFun are user-specified tolerances);

(ii) $i>$ MaxIter (user-specified maximum number of iterations);

(iii) the number of fine model evaluations exceeds a user-specified value;

(iv) $\delta^{(i)}<0.1 \cdot \operatorname{Tol} X$ (the TR radius is smaller than $10 \%$ of the argument tolerance). 
Note that $\overline{\boldsymbol{R}}_{f}$ is defined in such a way that $\overline{\boldsymbol{R}}_{f}\left(\boldsymbol{x}^{(i+1)}\right)=$ $\boldsymbol{R}_{f}\left(\boldsymbol{x}^{(i+1)}\right)$ if $\boldsymbol{x}^{(i+1)}=s\left(\boldsymbol{x}^{(i+1)}\right)$, i.e., if $\boldsymbol{x}^{(i+1)}$ is an on-grid point. The motivation behind this kind of interpolation follows from the fact that for each $i=1,2, \ldots, \boldsymbol{R}_{s}^{(i)}$ is defined using some or all of the information concerning the fine model gathered during the previous iterations. Thus, $R_{s}^{(i)}$ is the best available source of information about $\boldsymbol{R}_{f}$ in the neighborhood of $\boldsymbol{x}^{(i)}$. Note that (16) is not a global interpolation method. It is only local and tailored to work with SM optimization algorithms.

In order to use interpolation scheme (16), we need to modify the matching measure (10) in the following way:

$$
\begin{aligned}
& \varepsilon^{(i)}(\boldsymbol{A}, \boldsymbol{B}, \boldsymbol{c})=\sum_{k=0}^{i} w_{k} \| \boldsymbol{R}_{f}\left(s\left(\boldsymbol{x}^{(k)}\right)\right) \\
&-\boldsymbol{A} \cdot \boldsymbol{R}_{c}\left(B \cdot s\left(\boldsymbol{x}^{(k)}\right)+\boldsymbol{c}\right) \| \\
&+\sum_{k=0}^{i} v_{k} \| \overline{\boldsymbol{J}}_{\boldsymbol{R}_{f}}\left(s\left(\boldsymbol{x}^{(k)}\right)\right) \\
& \quad-\boldsymbol{A} \cdot \overline{\boldsymbol{J}}_{\boldsymbol{R}_{c}}\left(\boldsymbol{B} \cdot s\left(\boldsymbol{x}^{(k)}\right)+\boldsymbol{c}\right) \cdot \boldsymbol{B} \|
\end{aligned}
$$

where symbol $\overline{\boldsymbol{J}}_{\boldsymbol{R}_{f}}\left(\overline{\boldsymbol{J}}_{\boldsymbol{R}_{c}}\right)$ denotes an approximation of the fine (coarse) model Jacobian using finite differences with points lying on the grid. Although such estimation may be poor, especially for coarse grids, the use of Jacobian estimation in parameter extraction (17) may be beneficial in many cases, because the matching measure with $\overline{\boldsymbol{J}}_{\boldsymbol{R}_{f}}$ and $\overline{\boldsymbol{J}}_{\boldsymbol{R}_{c}}$ is, in a way, equivalent to multipoint parameter extraction at points corresponding to the finite difference scheme used for Jacobian approximation. On the other hand, the term $\boldsymbol{E}$ should not be used because its accuracy is essential for algorithm performance. For calculation of the matrix $\boldsymbol{d}^{(i)}$ we use, instead of (8), the new formula

$$
\boldsymbol{d}^{(i)}=\overline{\boldsymbol{R}}_{f}\left(\boldsymbol{x}^{(i)}\right)-\boldsymbol{A}^{(i)} \cdot \boldsymbol{R}_{c}\left(\boldsymbol{B}^{(i)} \cdot \boldsymbol{x}^{(i)}+\boldsymbol{c}^{(i)}\right) .
$$

One can also use an alternative matching measure based on the interpolated fine model response

$$
\begin{aligned}
\varepsilon^{(i)}(\boldsymbol{A}, \boldsymbol{B}, \boldsymbol{c}) \\
\quad \sum_{k=0}^{i} w_{k}\left\|\overline{\boldsymbol{R}}_{f}\left(\boldsymbol{x}^{(k)}\right)-\boldsymbol{A} \cdot \boldsymbol{R}_{c}\left(\boldsymbol{B} \cdot \boldsymbol{x}^{(k)}+\boldsymbol{c}\right)\right\| \\
\quad+\sum_{k=0}^{i} v_{k}\left\|\overline{\boldsymbol{J}}_{\boldsymbol{R}_{f}}\left(s\left(\boldsymbol{x}^{(k)}\right)\right)-\boldsymbol{A} \cdot \boldsymbol{J}_{\boldsymbol{R}_{c}}\left(\boldsymbol{B} \cdot \boldsymbol{x}^{(k)}+\boldsymbol{c}\right) \cdot \boldsymbol{B}\right\|
\end{aligned}
$$

which uses an approximated fine model response at $\boldsymbol{x}^{(k)}$ instead of the fine model response at iteration points snapped to the grid. Experiments indicate that this matching measure performs almost as well as (17); however, we will not use it further in this paper.
It should be emphasized that the proposed interpolation scheme uses already available fine model data — no additional fine model evaluations are necessary to perform this interpolation. The method is simple and straightforward to implement.

The SM optimization algorithm that uses the interpolation scheme introduced in this section is the same as (14) except that we use $f^{(i)}=U\left(\overline{\boldsymbol{R}}_{f}\left(\boldsymbol{x}^{(i)}\right)\right)$ to calculate $f^{(i)}$ in (12) while using our interpolation scheme, matching measure (17) instead of (10), and (18) instead of (8); also we use condition $\left\|\overline{\boldsymbol{R}}_{f}^{(i)}-\overline{\boldsymbol{R}}_{f}^{(i-1)}\right\| \leq$ TolFun as a convergence measure (instead of $\left\|\boldsymbol{R}_{f}^{(i)}-\boldsymbol{R}_{f}^{(i-1)}\right\| \leq$ TolFun). Using the interpolated fine model for calculating the gain ratio (12) as well as in the termination condition are the factors that affect the operation of the SM algorithm and allow us to obtain a better resolution and accuracy of SM optimization.

For further use, we also define a function $\tilde{R}_{f}: X_{f} \rightarrow R^{m}$, an extension of $\left.\boldsymbol{R}_{f}\right|_{\bar{X}_{f}}\left(\boldsymbol{R}_{f}\right.$ restricted to $\left.\bar{X}_{f}\right)$ onto $X_{f}$ such that

$$
\tilde{\boldsymbol{R}}_{f}(\boldsymbol{x})=\boldsymbol{R}_{f}(s(\boldsymbol{x}))
$$

i.e., $\tilde{\boldsymbol{R}}_{f}$ is the piece-wise constant extension of $\left.\boldsymbol{R}_{f}\right|_{\bar{X}_{f}}$ onto $X_{f}$ (which is equivalent to the actual fine model used while performing SM optimization without any interpolation scheme); $s$ is the snapping function defined by (15).

\section{EXAMPLES}

In this section, we describe results of numerical experiments conducted to verify the interpolation scheme proposed in Section III. We considered a couple of test problems, including synthetic examples (i.e., problems for which the fine model is defined on a continuous domain; however, we evaluate it only on a given grid - this allows us to perform reliable verification of the results) and real microwave problems in which the fine model is evaluated using commercial simulators and it really is available on a finite grid only (in this case, our results are verified by means of simulating the fine model with a very fine grid).

\section{A. Two-Section Capacitively Loaded Impedance Transformer}

As our first example, we used a two-section impedance transformer [21]. The "coarse" and "fine" models for the two-section impedance transformer are shown in Fig. 1. The values of the fine model capacitances are $C_{1 \ldots 3}=10 \mathrm{pF}$. The characteristic impedances are kept fixed at the optimal values $\left[\begin{array}{ll}Z_{1} & Z_{2}\end{array}\right]=\left[\begin{array}{ll}4.472 & 2.236\end{array}\right] \Omega$. The physical lengths $L_{1}$ and $L_{2}$ of the two transmission lines are selected as designable parameters. Twenty-one frequency points are simulated per sweep (uniformly distributed in the interval [0.5, 1.5] $\mathrm{GHz}$ ). We consider the input reflection coefficient response $f^{(i)}(\boldsymbol{x})=\left|S_{11}\left(\omega^{(j)} ; \boldsymbol{x}\right)\right|$ of both models which is a function of the real frequency $\omega$ and the designable parameters $\boldsymbol{x}=\left[\begin{array}{ll}L_{1} & L_{2}\end{array}\right]^{T}$. Both fine and coarse models are implemented in MATLAB. Design specifications are $\left|S_{11}\left(\omega^{(j)} ; \boldsymbol{x}\right)\right| \leq 0.5$ in the whole frequency range.

Optimization of the model was performed using our SM optimization algorithm with the trust region method. We used the surrogate model $\boldsymbol{A} \boldsymbol{B} \boldsymbol{c} \boldsymbol{d}_{a l l .1}$ (see Section II for details). Design 


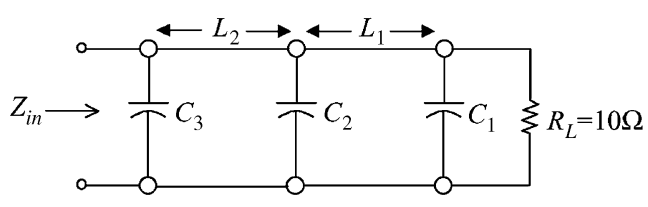

(a)

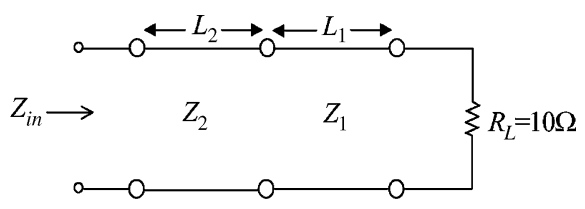

(b)

Fig. 1. (a) "Fine" and (b) "coarse" model, two-section capacitively loaded impedance transformer [21].

TABLE III

COMPARISON OF EXPERIMENTS ON THE TWO-SECTION TRANSFORMER TEST PROBLEM

\begin{tabular}{ccccc}
\hline $\begin{array}{c}\text { Grid } \\
\text { Size }\end{array}$ & Interpolation & $\begin{array}{c}\text { Specification }^{2} \\
\text { Error }^{2}\end{array}$ & $\begin{array}{c}\text { Accurate Spec. } \\
\text { Error }^{3}\end{array}$ & $\begin{array}{c}\text { Response } \\
\text { Accuracy }^{4}\end{array}$ \\
\hline 2 & No & -0.035 & -0.013 & 0.063 \\
2 & Yes & -0.043 & -0.042 & 0.016 \\
5 & No & -0.044 & -0.025 & 0.160 \\
5 & Yes & -0.043 & -0.042 & 0.048 \\
10 & No & -0.024 & -0.013 & 0.470 \\
10 & Yes & -0.043 & -0.043 & 0.047 \\
20 & No & 0.015 & 0.084 & 0.231 \\
20 & Yes & -0.053 & -0.042 & 0.136 \\
\hline \hline
\end{tabular}

$\overline{1}$ 'No' means that model (20) is used in the optimization procedure; 'Yes' means that we use interpolation scheme (16).

${ }^{2}$ specification error at the final iteration according to the currently used fine model (i.e., either model (20) if no interpolation is used, or model (16) if our interpolation scheme is utilized).

${ }^{3}$ specification error at the final iteration according to the exact fine model (i.e., the model available on a continuous domain).

${ }^{4} l_{2}$ norm of the difference between the response obtained using the current model (i.e., (20) or (16), respectively) and the exact response (model on a continuous domain).

variables are normalized to the coarse model optimal solution, i.e., the normalized starting point is $\boldsymbol{x}^{(0)}=[9090]^{T}$.

In our experiments we used four different grids: $2,5,10$, and 20 (i.e., we restrict evaluation of the fine model only to the points corresponding to the grid of a given size). We performed optimization of the two-section transformer using i) our interpolation scheme (16) and ii) no interpolation so that the fine model was evaluated at the nearest grid point regardless of the actual value of the coordinates [i.e., (20) was used]. Table III shows the details of our experiments, as well as the results, i.e., a comparison of the quality of solutions obtained with and without interpolation for different grid sizes. Fig. 2 shows the results for the grid size 20: the fine model response at the final iteration (interpolated and accurate) for optimization without interpolation [see Fig. 2(a)] and with the SM-based interpolation [see Fig. 2(b)]. It follows from the results presented in Table III that using our interpolation scheme gives satisfactory results even for the largest grid (a grid of 20 is more than $20 \%$ of the design parameter values at the final solution). The specification error according to interpolated model is reliable and response accuracy is good.

\section{B. Seven-Section Capacitively Loaded Impedance Transformer}

Our second example is a seven-section capacitively loaded impedance transformer [21]. The "coarse" and "fine" models for the seven-section impedance transformer are shown in Fig. 3. The load impedance is $100 \Omega$ and the line impedance is $50 \Omega$. The values of the fine model capacitances are $C_{1 \ldots 8}=0.025 \mathrm{pF}$. The characteristic impedances are kept

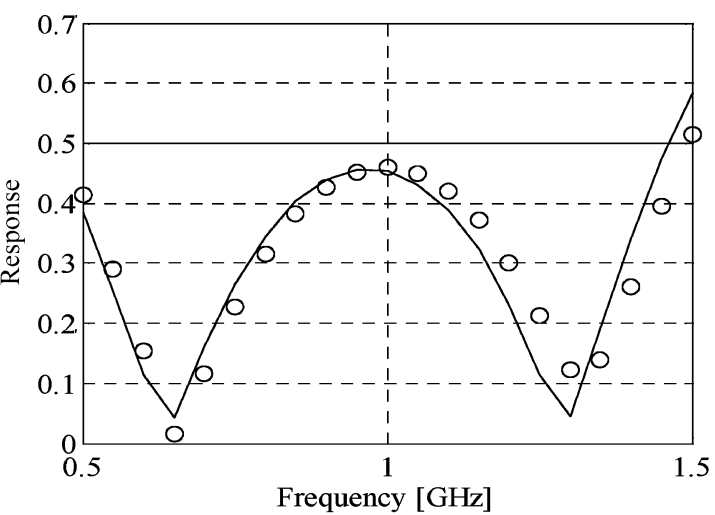

(a)

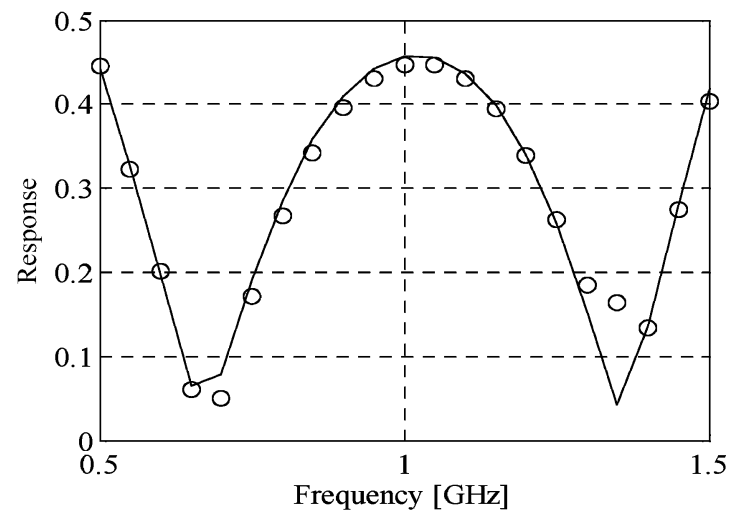

(b)

Fig. 2. Optimization results for grid size 20 for optimization: (a) without interpolation and (b) with interpolation: response at the final iteration according to the current model (points) and the exact response (i.e., obtained using the model available on a continuous domain) at the final iteration (solid line).

fixed at the optimal values $\left[\begin{array}{lllllll}Z_{1} & Z_{2} & Z_{3} & Z_{4} & Z_{5} & Z_{6} & Z_{7}\end{array}\right]=$ [91.9 85.5 78.2 70.7 64.0 58.4 54.3] $\Omega$. The physical lengths $L_{i}, i=1, \ldots, 7$, of the seven transmission lines are selected as designable parameters; 68 frequency points are simulated per sweep (uniformly distributed in the interval $[1.0,7.7] \mathrm{GHz}$ ).

Both fine and coarse models are implemented in MATLAB. We consider the input reflection coefficient response $f^{(i)}(\boldsymbol{x})=\left|S_{11}\left(\omega^{(j)} ; \boldsymbol{x}\right)\right|$ of both models which is a function of the real frequency $\omega$ and the designable parameters $\boldsymbol{x}=\left[\begin{array}{lllllll}L_{1} & L_{2} & L_{3} & L_{4} & L_{5} & L_{6} & L_{7}\end{array}\right]^{T}$. Design specifications are $\left|S_{11}\left(\omega^{(j)} ; \boldsymbol{x}\right)\right| \leq 0.07$ in the whole frequency range.

Optimization of the model was performed using our SM optimization algorithm with the trust region method. We used the surrogate model $\boldsymbol{A B} \boldsymbol{c d}_{4.1}$ (see Section II for details). Design variables are normalized to the coarse model optimal solution, i.e., the normalized starting point is $\boldsymbol{x}^{(0)}=\left[\begin{array}{lllllll}1 & 1 & 1 & 1 & 1 & 1 & 1\end{array}\right]^{T}$. 


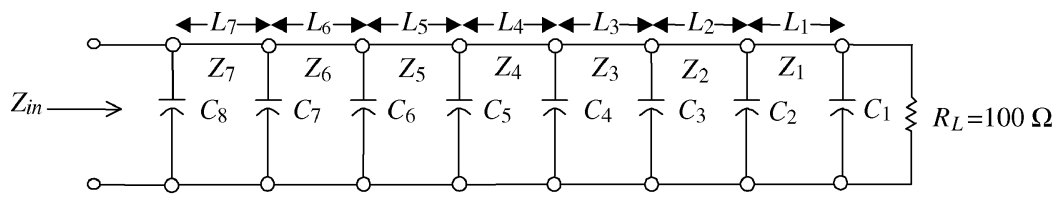

(a)

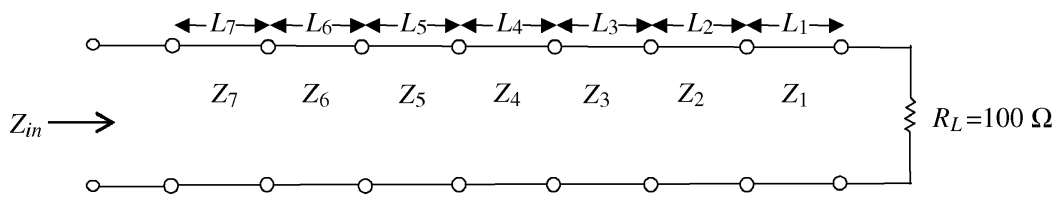

(b)

Fig. 3. Seven-section capacitively loaded impedance transformer. (a) "Fine" model. (b) "Coarse" model [21].

TABLE IV

COMPARISON OF EXPERIMENTS ON THE SEVEN-SECTION TRANSFORMER TEST PROBLEM

\begin{tabular}{ccccc}
\hline \hline $\begin{array}{l}\text { Grid } \\
\text { Size }\end{array}$ & Interpolation & $\begin{array}{c}\text { Specification } \\
\text { Error }^{2}\end{array}$ & $\begin{array}{c}\text { Accurate Spec. } \\
\text { Error }^{3}\end{array}$ & $\begin{array}{c}\text { Response } \\
\text { Accuracy }\end{array}$ \\
\hline 0.02 & No & -0.0054 & -0.0065 & 0.021 \\
0.02 & Yes & -0.0093 & -0.0091 & 0.002 \\
0.05 & No & -0.0027 & -0.0005 & 0.060 \\
0.05 & Yes & -0.0096 & -0.0092 & 0.005 \\
0.10 & No & 0.0015 & 0.0005 & 0.058 \\
0.10 & Yes & -0.0091 & -0.0084 & 0.009 \\
0.20 & No & 0.0345 & -0.0019 & 0.156 \\
0.20 & Yes & -0.0090 & -0.0050 & 0.027 \\
\hline \hline
\end{tabular}

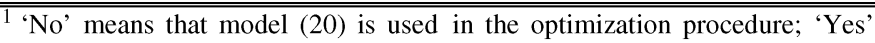
means that we use interpolation scheme (16).

${ }^{2}$ specification error at the final iteration according to the currently used fine model (i.e., either model (20) if no interpolation is used, or model (16) if our interpolation scheme is utilized).

${ }^{3}$ specification error at the final iteration according to the exact fine model (i.e., the model available on a continuous domain).

${ }^{4} l_{2}$ norm of the difference between the response obtained using the current model (i.e., (20) or (16), respectively) and the exact response (model on a continuous domain).

In our experiments we used four different grids: 0.02, 0.05, 0.10 , and 0.20 . We performed optimization of the seven-section transformer using i) an interpolation scheme (16) and ii) no interpolation, so that the fine model was evaluated at the nearest grid point regardless of the actual value of the coordinates [i.e., (20) was used]. Table IV shows the details of our experiments as well as the results, i.e., a comparison of the quality of solutions obtained with and without interpolation for different grid sizes. Fig. 4 shows the results for grid size 0.2: the fine model response at the final iteration (interpolated and accurate) for optimization without interpolation [see Fig. 4(a)] and with the SM-based interpolation [see Fig. 4(b)].

It follows from the results presented in Table IV that using our interpolation scheme gives satisfactory results even for relatively coarse grids (a grid of 0.10 is more than $10 \%$ of the design parameter values at the final solution). The specification error according to the interpolated model is reliable and the response accuracy is good. Even for a grid of 0.20, the response accuracy is acceptable.

\section{Three-Section Microstrip Transformer}

Our third test problem is the three-section microstrip impedance transformer shown in Fig. 5 [22]. The coarse

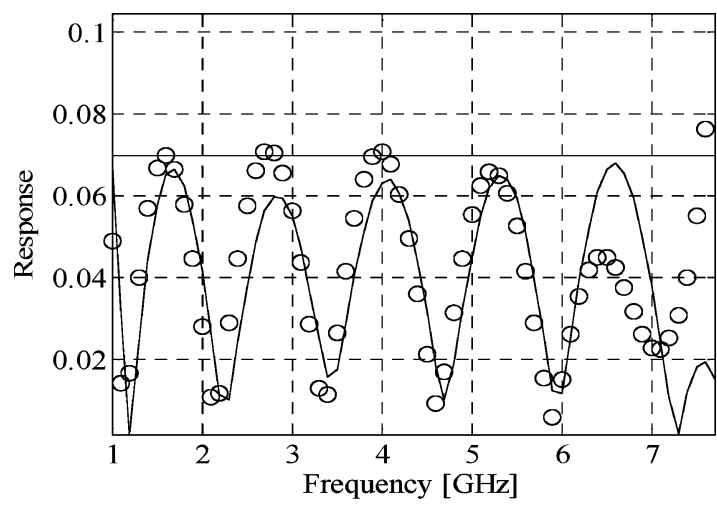

(a)

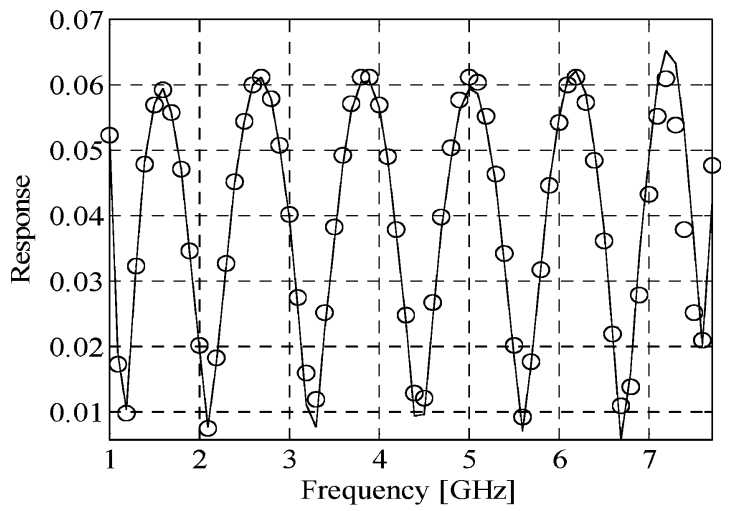

(b)

Fig. 4. Optimization results for grid size 0.2 for optimization: (a) without interpolation and (b) with interpolation: response at the final iteration according to the current model (points) and the exact response (i.e., obtained using the model available on a continuous domain) at the final iteration (solid line).

model is shown in Fig. 6. Well-known empirical formulas are used to express electrical parameters in terms of physical dimensions. The design specifications are $\left|S_{11}\right| \leq 0.09$ for $5 \mathrm{GHz} \leq \omega \leq 15 \mathrm{GHz}$. The designable parameters are the width and physical length of each microstrip line. Here, the reflection coefficient $S_{11}$ is used to match the two model responses. The fine model is a Sonnet $\boldsymbol{e m}$ [23] model. The designable parameters for the fine model are the widths and physical lengths of the three microstrip lines. The thickness of the dielectric substrate is $0.635 \mathrm{~mm}$ ( $25 \mathrm{mil})$ and its relative permittivity is 9.7 . The effect of nonideal dielectric is considered 


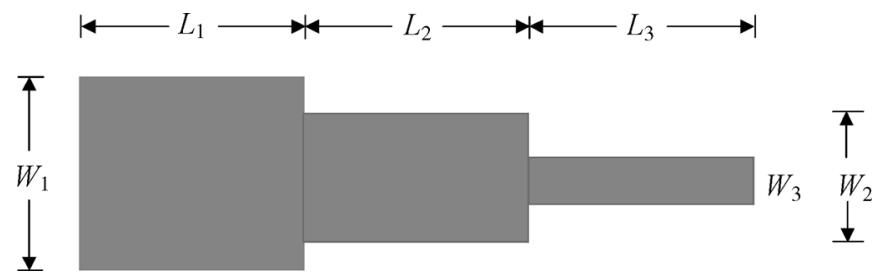

Fig. 5. Three-section 3:1 microstrip impedance transformer: structure and dimensions [22].

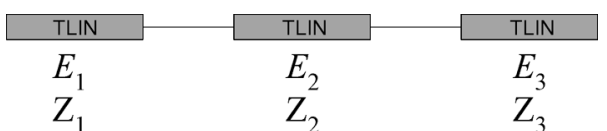

Fig. 6. Three-section 3:1 microstrip impedance transformer: coarse model [22].

by setting the loss tangent to 0.002 . We use 11 frequency points in the sweep. The coarse model is implemented in MATLAB.

Optimization of the model was performed using an our SM optimization algorithm with a trust region method. We used the surrogate model $\boldsymbol{A B} \boldsymbol{B} \boldsymbol{d}_{\text {all. } .0}$ (see Section II). The starting point is $\boldsymbol{x}^{(0)}=\left[\begin{array}{lllll}117 & 121 & 124 & 15 & 62\end{array}\right]^{T}$ (coarse model optimal solution).

In our experiments we used three different grids: grid $1-\mathrm{a}$ horizontal grid of $1 \mathrm{~mm}$, a vertical grid of $0.1 \mathrm{~mm}$; grid 2-a horizontal grid of $4 \mathrm{~mm}$, a vertical grid of $0.2 \mathrm{~mm}$; and grid 3 -a horizontal grid of $10 \mathrm{~mm}$, a vertical grid of $0.25 \mathrm{~mm}$. We performed optimization of the three-section transformer using: 1) our interpolation scheme (16) and 2) no interpolation, so that the fine model was evaluated at the nearest grid point regardless of the actual value of the coordinates [i.e., (20) was used].

Since this is a real (not synthetic) example, the accuracy of the results was verified by simulating the results using a fine grid with the following parameters: a horizontal grid of $0.2 \mathrm{~mm}$ and a vertical grid of $0.01 \mathrm{~mm}$. Simulation results obtained using this grid were treated as exact (accurate) responses of the fine model.

Table V shows the details of our experiments, as well as the results, i.e., a comparison of the quality of solutions obtained with and without interpolation for different grid sizes. Fig. 7 shows the results for grid size 10/0.25: the fine model response at the final iteration (interpolated and accurate) for optimization without interpolation [see Fig. 7(a)] and with the SM-based interpolation [see Fig. 7(b)].

It is seen from the results in Table V that our interpolation scheme gives satisfactory results for all grids. Specification error according to the interpolated model is reliable and the response accuracy is good.

\section{Six-Section H-Plane Waveguide Filter}

Our last test problem is the six-section $H$-plane waveguide filter shown in Fig. 8 [24]. We use a waveguide of width 1.372 in $(34.85 \mathrm{~mm})$. The six waveguide sections are separated by seven $H$-plane septa, which have a finite thickness of 0.0245 in $(0.6223 \mathrm{~mm})$. Design parameters are the section lengths $L_{1}, L_{2}$, and $L_{3}$ and the septa widths $W_{1}, W_{2}, W_{3}$, and $W_{4}$. We use 51 points from $5.0 \mathrm{GHz} \leq \omega \leq 10.0 \mathrm{GHz}$ in the frequency sweep. The design specifications are $\left|S_{11}\right| \leq 0.16$ for $5.4 \mathrm{GHz} \leq \omega \leq$
TABLE V

COMPARISON OF EXPERIMENTS ON THE THREE-SECTION TRANSFORMER TEST PROBLEM

\begin{tabular}{ccccc}
\hline \hline $\begin{array}{c}\text { Grid } \\
\text { Size }\end{array}$ & Interpolation $^{1}$ & $\begin{array}{c}\text { Specification } \\
\text { Error }^{2}\end{array}$ & $\begin{array}{c}\text { Accurate Spec. } \\
\text { Error }^{3}\end{array}$ & $\begin{array}{c}\text { Response } \\
\text { Accuracy }^{4}\end{array}$ \\
\hline $1 / 0.1$ & No & 0.001 & 0.004 & 0.014 \\
$1 / 0.1$ & Yes & -0.006 & -0.005 & 0.006 \\
$4 / 0.2$ & No & 0.003 & 0.006 & 0.023 \\
$4 / 0.2$ & Yes & -0.005 & -0.002 & 0.011 \\
$10 / 0.25$ & No & 0.020 & 0.026 & 0.020 \\
$10 / 0.25$ & Yes & -0.006 & -0.005 & 0.009 \\
\hline \hline
\end{tabular}

1'No' means that model (20) is used in the optimization procedure; 'Yes' means that we use interpolation scheme (16).

${ }^{2}$ specification error at the final iteration according to the currently used fine model (i.e., either model (20) if no interpolation is used, or model (16) if our interpolation scheme is utilized).

${ }^{3}$ specification error at the final iteration according to the exact fine model (i.e., the verification of the fine model using a very fine grid).

${ }^{4} l_{2}$ norm of the difference between the response obtained using the current model (i.e., (20) or (16), respectively) and the exact response (model using a very fine grid).

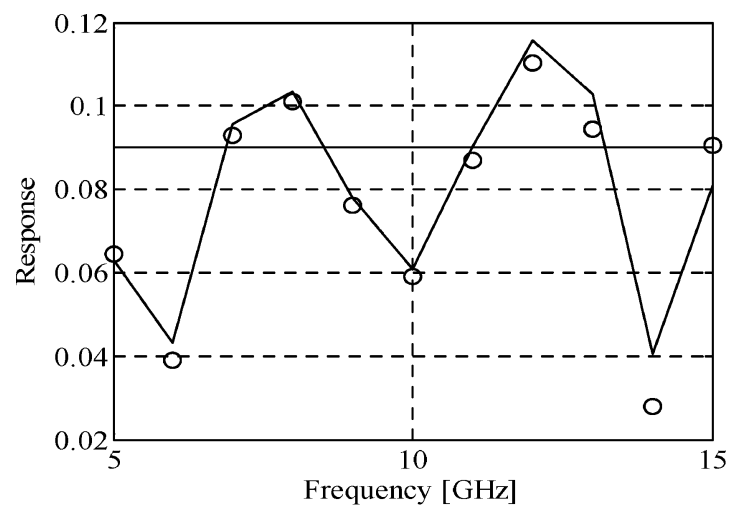

(a)

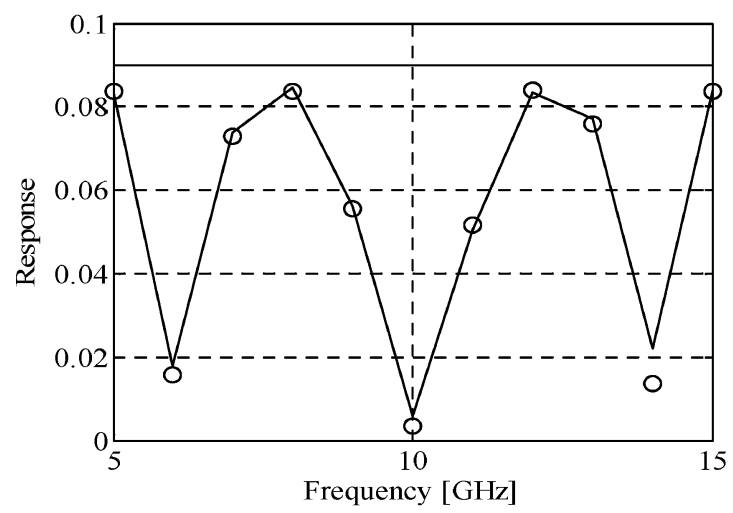

(b)

Fig. 7. Optimization results for grid size $10 / 0.25$ for optimization: (a) without interpolation and (b) with interpolation: response at the final iteration according to the current model (points) and the exact response (i.e., obtained using the model available on a very fine grid) at the final iteration (solid line).

$9.0 \mathrm{GHz},\left|S_{11}\right| \geq 0.85$ for $5.0 \mathrm{GHz} \leq \omega \leq 5.2 \mathrm{GHz}$, and $\left|S_{11}\right| \geq 0.5$ for $9.5 \mathrm{GHz} \leq \omega \leq 10.0 \mathrm{GHz}$.

The fine model is simulated using MEFiSTo [25] in a two-dimensional mode. The MATLAB coarse model (Fig. 9) has lumped inductances and dispersive transmission line sections. We simplify formulas due to Marcuvitz for the inductive susceptances corresponding to the $H$-plane septa. 


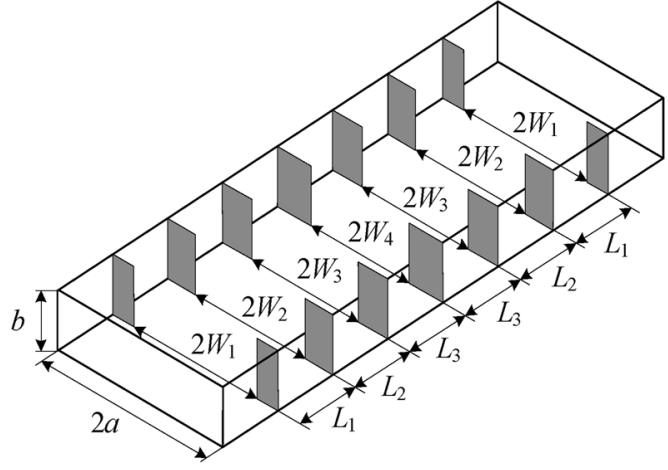

Fig. 8. Six-section $H$-plane waveguide filter: the three-dimensional view [24].

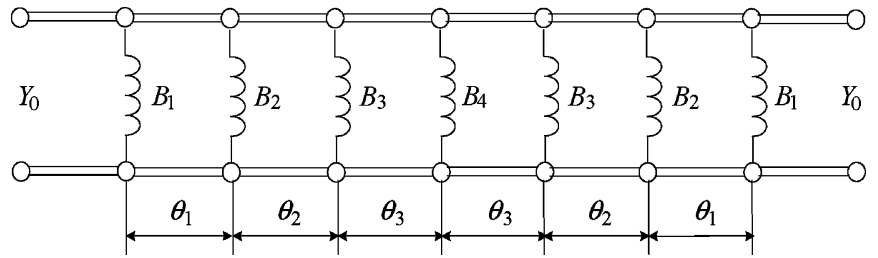

Fig. 9. Six-section $H$-plane waveguide filter: the equivalent empirical circuit model [24].

Optimization of the model was performed using our SM optimization algorithm with a trust region method. We used the surrogate model $\boldsymbol{A B c d _ { \text { all. } }}$ (see Section II for details). The starting point is

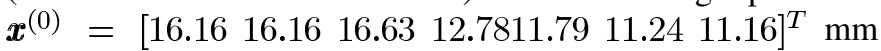
(coarse model optimal solution).

In our experiments we used four different grids: 0.5, 1.0, 2.0, and $4.0[\mathrm{~mm}]$. We performed optimization of the six-section $H$-plane waveguide filter using i) our interpolation scheme (16) and ii) no interpolation so that the fine model was evaluated at the nearest grid point regardless of the actual value of the coordinates [i.e., (20) was used]. Accuracy of the results was verified by the MEFiSTo simulator with its rubber-cell feature, which allows evaluation of the model at any point: on-grid or off-grid.

Table VI shows the details of our experiments, as well as the results, i.e., a comparison of the quality of the solutions obtained with and without interpolation for different grid sizes. Fig. 10 shows the results for grid size 4: the fine model response at the final iteration (interpolated and accurate) for optimization without interpolation [see Fig. 10(a)] and with the SM-based interpolation [see Fig. 10(b)].

It is seen from the results that our interpolation scheme gives satisfactory results for all grids. The specification error according to our interpolated model is reliable. Accuracy of the response is not very good, which is mainly because of the very steep response of the filter at the edge of the stopband.

\section{MATHEMATiCAL Motivation OF THE SM INTERPOLATION SCHEME}

The results of Section IV can be accounted for using the simple error estimation shown below. As before, let $\tilde{\boldsymbol{R}}_{f}: X_{f} \rightarrow$
TABLE VI

COMPARISON OF EXPERIMENTS ON THE $H$-PLANE WAVEGUIDE FILTER TEST PROBLEM

\begin{tabular}{ccccc}
\hline \hline $\begin{array}{c}\text { Grid } \\
\text { Size }\end{array}$ & Interpolation & $\begin{array}{c}\text { Specification } \\
\text { Error }^{2}\end{array}$ & $\begin{array}{c}\text { Accurate Spec. } \\
\text { Error }^{3}\end{array}$ & $\begin{array}{c}\text { Response } \\
\text { Accuracy }^{4}\end{array}$ \\
\hline 0.5 & No & 0.044 & 0.025 & 0.196 \\
0.5 & Yes & -0.039 & 0.055 & 0.181 \\
1.0 & No & 0.118 & 0.040 & 0.472 \\
1.0 & Yes & -0.039 & 0.020 & 0.159 \\
2.0 & No & 0.421 & 0.186 & 1.219 \\
2.0 & Yes & -0.040 & -0.018 & 0.189 \\
4.0 & No & 0.541 & 0.184 & 1.509 \\
4.0 & Yes & -0.043 & -0.011 & 0.161 \\
\hline \hline
\end{tabular}

1 'No' means that model (20) is used in the optimization procedure; 'Yes' means that we use interpolation scheme (16).

${ }^{2}$ specification error at the final iteration according to the currently used fine model (i.e., either model (20) if no interpolation is used, or model (16) if our interpolation scheme is utilized).

${ }^{3}$ specification error at the final iteration according to the exact fine model (i.e., the model available on a continuous domain).

${ }^{4} l_{2}$ norm of the difference between the response obtained using the current model (i.e., (20) or (16), respectively) and the exact response (model on a continuous domain).

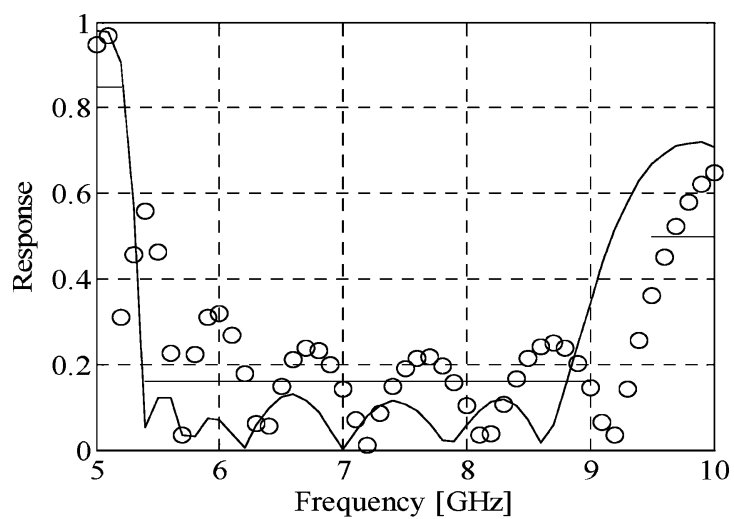

(a)

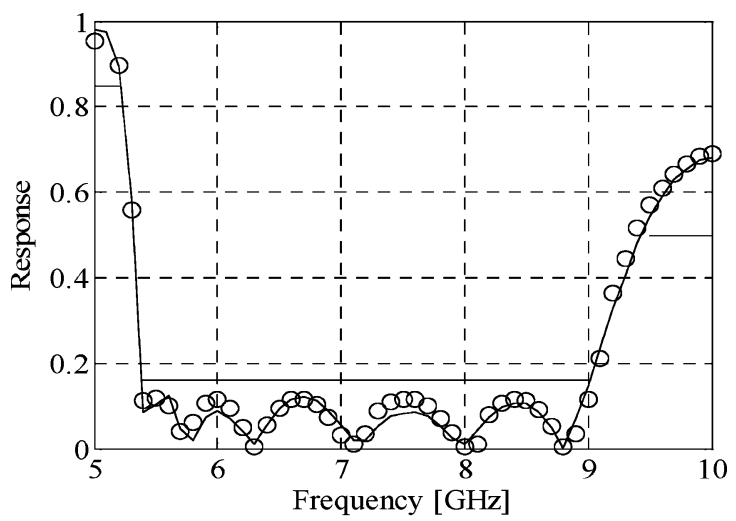

(b)

Fig. 10. Optimization results for grid size 4 for optimization: (a) without interpolation and (b) with interpolation: response at the final iteration according to the current model (points) and the exact response (i.e., obtained using the model available on a continuous domain) at the final iteration (solid line).

$R^{m}$ denote the piece-wise constant extension of $\left.\boldsymbol{R}_{f}\right|_{\bar{X}_{f}}$ onto $X_{f}$ defined by (20) and $\overline{\boldsymbol{R}}_{f}$ be the interpolated fine model defined by (16). Let $\boldsymbol{R}_{s . \boldsymbol{x}^{0}}: X_{f} \rightarrow R^{m}$ be a surrogate model of $\boldsymbol{R}_{f}$ defined in the neighborhood of $\boldsymbol{x}^{0} \in \bar{X}_{f}$ (i.e., $\boldsymbol{x}^{0}$ is on grid) and 


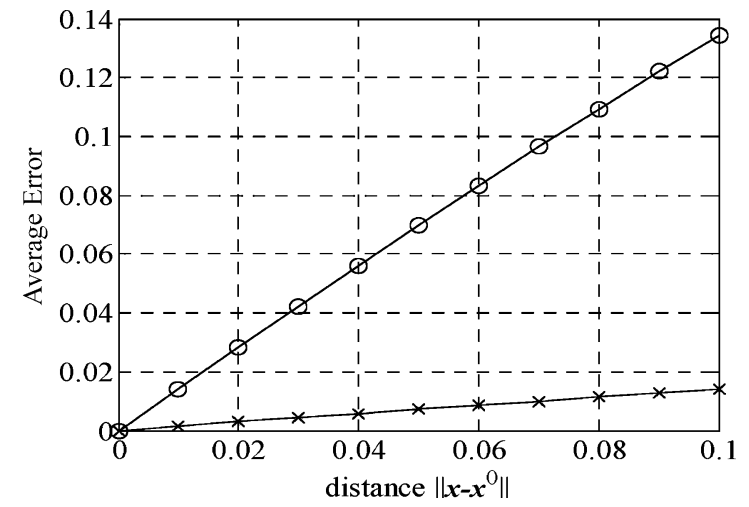

Fig. 11. $l_{2}$ error between the fine model $\boldsymbol{R}_{f}$ and models $\tilde{\boldsymbol{R}}_{f}$ (points) and $\overline{\boldsymbol{R}}_{f}$ (crosses) versus the distance from the reference point for the seven-section transformer.

$D_{\boldsymbol{x}^{0}}=\left\{\boldsymbol{x} \in X_{f}: \forall \boldsymbol{z} \in \bar{X}_{f} \backslash\left\{\boldsymbol{x}^{0}\right\}\left\|\boldsymbol{x}-\boldsymbol{x}^{0}\right\| \leq\|\boldsymbol{x}-\boldsymbol{z}\|\right\}$ (i.e., the set of all points in $X_{f}$ that are closer to $x^{0}$ than to any other grid point). Using the mean value theorem, we obtain the following estimates:

$$
\left\|\boldsymbol{R}_{f}(\boldsymbol{x})-\tilde{\boldsymbol{R}}_{f}(\boldsymbol{x})\right\| \leq C_{1}\left\|\boldsymbol{x}-\boldsymbol{x}^{0}\right\|
$$

where

$$
C_{1}=\max _{\boldsymbol{x} \in D_{\boldsymbol{x}^{0}}}\left\|\boldsymbol{J}_{\boldsymbol{R}_{f}}(\boldsymbol{x})\right\|
$$

and

$$
\left\|\boldsymbol{R}_{f}(\boldsymbol{x})-\overline{\boldsymbol{R}}_{f}(\boldsymbol{x})\right\| \leq C_{2}\left\|\boldsymbol{x}-\boldsymbol{x}^{0}\right\|
$$

where

$$
C_{2}=\max _{\boldsymbol{x} \in D_{\boldsymbol{x}^{0}}}\left\|\boldsymbol{J}_{\boldsymbol{R}_{f}}(\boldsymbol{x})-\boldsymbol{J}_{\boldsymbol{R}_{s \cdot \boldsymbol{x}^{0}}}(\boldsymbol{x})\right\| .
$$

It follows from (21)-(24) that the interpolation error grows linearly with distance from $\boldsymbol{x}^{0}$ for both $\tilde{\boldsymbol{R}}_{f}$ and $\overline{\boldsymbol{R}}_{f}$. However, constant $C_{2}$ is in practice much smaller than $C_{1}$ because $\boldsymbol{R}_{s . x^{0}}$ is defined in such a way that it aims at reducing misalignment between $\boldsymbol{R}_{f}$ and the coarse model in the neighborhood of $\boldsymbol{x}^{0}$.

As an example, consider the seven-section transformer. Fig. 11 shows the average $l_{2}$ error between the fine model $\boldsymbol{R}_{f}$ and models $\tilde{\boldsymbol{R}}_{f}$ (points) and $\overline{\boldsymbol{R}}_{f}$ (crosses) versus the distance from the reference point (here $\boldsymbol{x}^{0}=\left[\begin{array}{lllllll}1 & 1 & 1 & 1 & 1 & 1 & 1\end{array}\right]^{T}$ ). The surrogate was set up using 15 points located in a star distribution with step 0.05 . The average was taken over 500 random points (separate statistics for each distance). It follows that, in this case, interpolation using the surrogate model reduces the error by a factor of ten. A similar experiment was carried out for the two-section transformer. In this case, interpolation using the surrogate model reduces the matching error by a factor of six.

The other aspect of SM interpolation is that it makes the optimization algorithm more stable. Without interpolation, the optimized function is piece-wise constant and thus highly discontinuous at boundary points between different subdomains $\boldsymbol{D}_{\boldsymbol{x}}$. Interpolation helps reduce these discontinuities. Fig. 12(a) shows

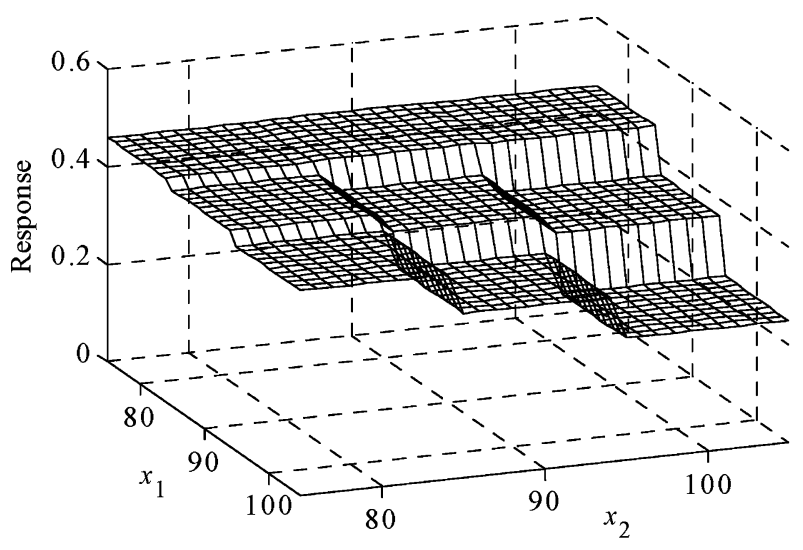

(a)

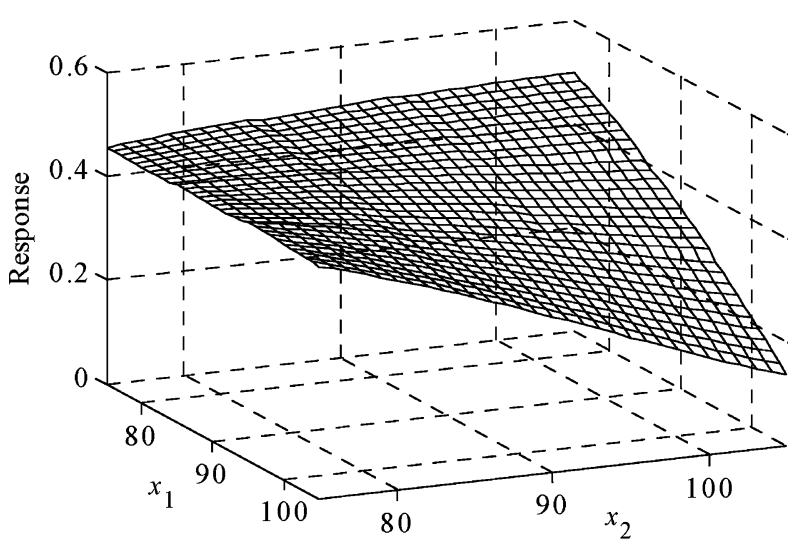

(b)

Fig. 12. Two-section transformer fine model response at $1 \mathrm{GHz}$ (normalized grid size ten): (a) without interpolation and (b) with SM-based interpolation.

the uninterpolated fine model response of the two-section transformer for frequency $1 \mathrm{GHz}$ with a grid equal to ten (in both directions). Fig. 12(b) shows the corresponding response for the model with interpolation. As a consequence, interpolation improves the convergence properties an SM algorithm. We omit the details.

\section{USING SM INTERPOLATION TO SPEED UP THE OPTIMIZATION PROCESS}

So far, we have shown that space-mapping-based interpolation allows us to perform optimization of models available on a given grid as if they are available on a continuous domain. In this section, we show that SM-based interpolation can be used to speed up the optimization process. The key features of the proposed interpolation scheme that allow this speedup are: no additional fine model evaluations required to perform interpolation and good reliability even for relatively coarse grids. Because of these features, instead of performing optimization using a fine grid (adjusted according to the required resolution), one can use SM-based interpolation and a much coarser grid to get a solution of similar resolution and quality in a shorter time.

For demonstration purposes, we use one of the examples described in Section IV: the three-section impedance transformer. In Experiment 1, we performed optimization using a horizontal grid of $1 \mathrm{~mm}$ and a vertical grid of $0.1 \mathrm{~mm}$ without interpolation 
TABLE VII

COMPARISON OF EXPERIMENTS ON THE THREE-SECTION TRANSFORMER TEST PROBLEM

\begin{tabular}{cccccccc}
\hline \hline $\begin{array}{c}\text { Experi- } \\
\text { ment } \\
\text { Number }\end{array}$ & $\begin{array}{c}\text { Grid } \\
\text { Size }\end{array}$ & $\begin{array}{c}\text { Interpo- } \\
\text { lation }\end{array}$ & $\begin{array}{c}\text { Fine } \\
\text { Model } \\
\text { Evals }\end{array}$ & $\begin{array}{c}\text { Relative } \\
\text { Optimiz. } \\
\text { Time }^{1}\end{array}$ & $\begin{array}{c}\text { Spec. } \\
\text { Error }^{2}\end{array}$ & $\begin{array}{c}\text { Accurate } \\
\text { Specification } \\
\text { Error }^{3}\end{array}$ & $\begin{array}{c}\text { Response } \\
\text { Accuracy }\end{array}$ \\
\hline 1 & $1 / 0.1$ & No & 4 & 1.000 & 0.001 & 0.004 & 0.014 \\
2 & $4 / 0.2$ & Yes & 3 & 0.094 & -0.005 & -0.002 & 0.011 \\
3 & $10 / 0.25$ & Yes & 5 & 0.050 & -0.006 & -0.005 & 0.009 \\
\hline \hline
\end{tabular}

${ }^{1}$ optimization time normalized with respect to Experiment 1.

${ }^{2}$ specification error at the final iteration according to the currently used fine model (i.e., either model (20) if no interpolation is used, or model (16) if our interpolation scheme is utilized).

${ }^{3}$ specification error at the final iteration according to the exact fine model (i.e., the verification of the fine model using a very fine grid).

${ }^{4} l_{2}$ norm of the difference between the response obtained using the current model (i.e., (20) or (16), respectively) and the exact response (model using a very fine grid).

assuming that this grid gives a satisfactory resolution. In Experiment 2, the three-section transformer was optimized using a horizontal grid of $4 \mathrm{~mm}$ and a vertical grid of $0.2 \mathrm{~mm}$ (with SM-based interpolation); in Experiment 3 we used a horizontal grid of $10 \mathrm{~mm}$ and a vertical grid of $0.25 \mathrm{~mm}$ (also with interpolation). The accuracy of the results was verified by evaluating the fine model with a very fine grid (horizontal of $0.2 \mathrm{~mm}$ and vertical of $0.01 \mathrm{~mm}$ ).

It follows from the results in Table VII that using interpolation indeed allows us to obtain significant reduction of optimization time. Moreover, the quality of the solution obtained with coarser grids and interpolation is better both with respect to the specification error and response accuracy.

It should be pointed out that the proposed interpolation scheme can be used to speed up the optimization process even if the fine model is available on a continuous domain. In particular, the user can introduce a so-called simulation grid, i.e., to limit the algorithm to evaluate the fine model only on this grid. This concept has already been used in commercial software, e.g., Empipe [26]. When the model is available only on a grid, we can use a coarser grid as a simulation grid. After some initial iterations, as the algorithm is about to converge, most of the subsequent iteration points will be snapped to the same grid point while evaluating the fine model. Provided that the fine model data is stored in the database, subsequent fine model evaluations cost virtually nothing (regardless of how many iterations are necessary to complete the optimization).

\section{ROBUSTNESS OF THE INTERPOLATION SCHEME}

It is natural to expect that the quality of the interpolation scheme (16) proposed in this paper depends on the quality of the coarse model used in the space mapping optimization procedure. This is because we expect that accuracy of the surrogate model is a function of the accuracy of the coarse model itself. However, our interpolation scheme is tightly connected with the space mapping algorithm so that the question "How robust is the interpolation?" is in fact "How robust is the space mapping algorithm itself?"

In order to get insight into this issue, we consider again the two-section impedance transformer example and examine the quality of the interpolation versus the quality of the coarse model. Let $R_{c}^{0}$ denote the original coarse model of the two-sec-

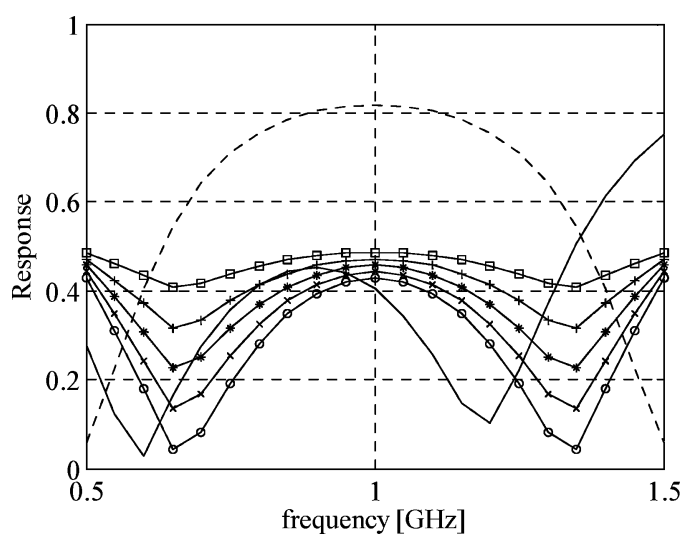

Fig. 13. Fine model response (solid line) and coarse model responses $\boldsymbol{R}_{c}^{0.0}$ (o), $\boldsymbol{R}_{c}^{0.2}(\times), \boldsymbol{R}_{c}^{0.4}(*), \boldsymbol{R}_{c}^{0.6}(+), \boldsymbol{R}_{c}^{0.8}(\square)$, and $\boldsymbol{R}_{c}^{Z}(--)$ at $\boldsymbol{x}^{(0)}=[9090]^{T}$.

tion transformer used in Section IV. We shall consider the family of coarse models $\boldsymbol{R}_{c}^{\alpha}$ defined as follows:

$$
\boldsymbol{R}_{c}^{\alpha}(\boldsymbol{x})=(1-\alpha) \cdot \boldsymbol{R}_{c}^{0}(\boldsymbol{x})+\alpha \cdot \boldsymbol{R}^{0}
$$

where $\boldsymbol{R}^{0}=[0.50 .5, \ldots, 0.5]^{T}$ is a constant vector. In other words, $\boldsymbol{R}_{c}^{\alpha}$ is a convex combination of the original coarse model and a constant vector. We also consider another coarse model $R_{q c}^{Z}$, which is the same as the original coarse model except that its characteristic impedances are the same and equal to the average of their optimal values, i.e., $Z_{1}=Z_{2}=3.354 \Omega$. Fig. 13 shows the fine model response and coarse model responses for models $\boldsymbol{R}_{c}^{\alpha}, \alpha=0,0.2,0.4,0.6,0.8$, and $\boldsymbol{R}_{c}^{Z}$ at $\boldsymbol{x}^{(0)}=[9090]^{T}$. It is seen that the coarse model becomes worse and worse with increasing $\alpha$. The model $\boldsymbol{R}_{c}^{Z}$ is the worst of all the models considered here. Note also that even the original coarse model is far from good.

Now, for each of these coarse models, we perform optimiza-

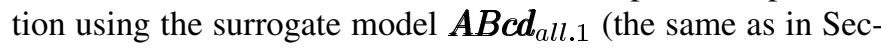
tion IV), imposing the grid of size 10 and using interpolation scheme (16). For comparison, we also perform SM optimization without a grid (so that we can see how the regular SM algorithm performs with increasingly bad coarse models). Table VIII shows the results, including specification errors and response accuracy.

The results in Table VIII indicate that our interpolation scheme provides good results for values of $\alpha$ up to 0.4 . For larger values of $\alpha$, response accuracy is no longer satisfactory. Thus, the interpolation is robust enough to work properly even with bad models, although it eventually fails when the coarse model is really bad. It follows from the last column of Table VIII, which provides the optimization results without using any grid (i.e., regular SM optimization with the fine model available on a continuous domain), that regular SM optimization begins to fail for about the same value of $\alpha$ (larger than 0.4).

This confirms what we said at the beginning of this section. Our interpolation scheme is tightly connected to the SM optimization algorithm, and if the coarse model becomes too bad for the SM optimization, it also becomes bad for SM interpolation. This is further confirmed by the results concerning the $\boldsymbol{R}_{c}^{Z}$ model: in this case both regular SM optimization (no grid, no interpolation) and SM optimization with grid and interpolation fail. 
TABLE VIII

Results FOR THE TwO-SECTION TRANSFORMER TEST PROBleM (TESTING ROBUSTNESS OF INTERPOLATION)

\begin{tabular}{ccccc}
\hline \hline $\begin{array}{c}\text { Mode } \\
\mathbf{l}\end{array}$ & $\begin{array}{c}\text { Specification } \\
\text { Error }^{1}\end{array}$ & $\begin{array}{c}\text { Accurate } \\
\text { Spec. Error }^{2}\end{array}$ & $\begin{array}{c}\text { Response } \\
\text { Accuracy }^{3}\end{array}$ & $\begin{array}{c}\text { Spec. Error For Regular SM } \\
\text { Optimization (No Grid) }\end{array}$ \\
\hline $\boldsymbol{R}_{c}^{0.0}$ & -0.043 & -0.043 & 0.047 & -0.043 \\
$\boldsymbol{R}_{c}^{0.2}$ & -0.042 & -0.043 & 0.063 & -0.043 \\
$\boldsymbol{R}_{c}^{0.4}$ & -0.041 & -0.043 & 0.088 & -0.042 \\
$\boldsymbol{R}_{c}^{0.6}$ & -0.041 & -0.029 & 0.277 & -0.039 \\
$\boldsymbol{R}_{c}^{0.8}$ & -0.033 & 0.018 & 0.329 & -0.030 \\
$\boldsymbol{R}_{c}^{Z}$ & 0.018 & 0.298 & 0.379 & 0.253 \\
\hline
\end{tabular}

$\overline{{ }^{1} \text { specification error at the final iteration according to the currently used fine }}$ model (i.e., either model (20) if no interpolation is used, or model (16) if our interpolation scheme is utilized)

${ }^{2}$ specification error at the final iteration according to the exact fine model (i.e., the model available on a continuous domain).

${ }^{3} l_{2}$ norm of the difference between the response obtained using the current model (i.e., (20) or (16), respectively) and the exact response (model on a continuous domain).

\section{CONCLUSION}

A novel space-mapping-based interpolation scheme has been presented. It is designed to work in conjunction with SM optimization algorithms. The method is useful if the fine model (the one that is supposed to be optimized) is available only on a finite grid. It allows us to estimate the response of the fine model at off-grid points and, as a result, increases the resolution of the design variable domain search and improves the quality of the fine model solution found by the optimization algorithm. Moreover, it can be used to speed up the entire optimization process by relaxing the grid requirements. The proposed method requires little computational effort; in particular, no additional fine model evaluations are necessary. Several examples presented in this paper verify the accuracy and robustness of our approach.

\section{REFERENCES}

[1] J. W. Bandler, R. M. Biernacki, S. H. Chen, P. A. Grobelny, and R. H. Hemmers, "Space mapping technique for electromagnetic optimization," IEEE Trans. Microw. Theory Tech., vol. 44, no. 12, pp. 536-544, Dec. 1994.

[2] J. W. Bandler, R. M. Biernacki, S. H. Chen, R. H. Hemmers, and K. Madsen, "Electromagnetic optimization exploiting aggressive space mapping," IEEE Trans. Microw. Theory Tech., vol. 43, no. 12, pp. 2874-2882, Dec. 1995

[3] J. W. Bandler, Q. S. Cheng, N. K. Nikolova, and M. A. Ismail, "Implicit space mapping optimization exploiting preassigned parameters," IEEE Trans. Microw. Theory Tech., vol. 52, no. 1, pp. 378-385, Jan. 2004.

[4] J. W. Bandler, Q. S. Cheng, D. H. Gebre-Mariam, K. Madsen, F. Pedersen, and J. Søndergaard, "EM-based surrogate modeling and design exploiting implicit, frequency and output space mappings," in IEEE MTT-S Int. Microw. Symp. Dig., Philadelphia, PA, Jun. 2003, pp. 1003-1006.

[5] J. W. Bandler, Q. S. Cheng, S. A. Dakroury, A. S. Mohamed, M. H. Bakr, K. Madsen, and J. Sondergaard, "Space mapping: the state of the art," IEEE Trans. Microw. Theory Tech., vol. 52, no. 1, pp. 337-361, Jan. 2004.

[6] S. J. Leary, A. Bhaskar, and A. J. Keane, "A constraint mapping approach to the structural optimization of an expensive model using surrogates," Opt. Eng., vol. 2, pp. 385-398, Dec. 2001.

[7] M. Redhe and L. Nilsson, "Using space mapping and surrogate models to optimize vehicle crashworthiness design," presented at the 9th AIAA/ISSMO Multidisciplinary Anal. Opt. Symp., Atlanta, GA, Sep. 2002, paper AIAA-2002-5536.
[8] H.-S. Choi, D. H. Kim, I. H. Park, and S. Y. Hahn, "A new design technique of magnetic systems using space mapping algorithm," IEEE Trans. Magn., vol. 37, no. 5, pp. 3627-3630, Sep. 2001.

[9] J. W. Bandler, M. A. Ismail, and J. E. Rayas-Sanchez, "Expanded space-mapping EM-based design framework exploiting preassigned parameters," IEEE Trans. Circuits Syst. I, vol. 49, no. 12, pp. 1833-1838, Dec. 2002.

[10] S. Koziel, J. W. Bandler, A. S. Mohamed, and K. Madsen, "Enhanced surrogate models for statistical design exploiting space mapping technology," in IEEE MTT-S Int. Microw. Symp. Dig., Long Beach, CA, Jun. 2005, pp. 1609-1612.

[11] J. W. Bandler, Q. S. Cheng, and S. Koziel, "Implementable space mapping approach to enhancement of microwave device models," in IEEE MTT-S Int. Microw. Symp. Dig., Long Beach, CA, Jun. 2005, pp. $1139-1142$.

[12] S. Koziel, J. W. Bandler, and K. Madsen, "Towards a rigorous formulation of the space mapping technique for engineering design," in Proc. Int. Symp. Circuits, Syst. (ISCAS), Kobe, Japan, May 2005, pp. 5605-5608.

[13] M. H. Bakr, J. W. Bandler, M. A. Ismail, J. E. Rayas-Sanchez, and Q.-J. Zhang, "Neural space-mapping optimization for EM-based design," IEEE Trans. Microw. Theory Tech., vol. 48, no. 12, pp. 2307-2315, Dec. 2000.

[14] V. K. Devabhaktuni, B. Chattaraj, M. C. E. Yagoub, and Q.-J. Zhang, "Advanced microwave modeling framework exploiting automatic model generation, knowledge neural networks, and space mapping," IEEE Trans. Microw. Theory Tech., vol. 51, no. 7, pp. 1822-1833, Jul. 2003.

[15] J. E. Rayas-Sanchez, "EM-based optimization of microwave circuits using artificial neural networks: the state-of-the-art," IEEE Trans. Microw. Theory Tech., vol. 52, no. 1, pp. 420-435, Jan. 2004.

[16] J. E. Rayas-Sanchez, F. Lara-Rojo, and E. Martinez-Guerrero, "A linear inverse space-mapping (LISM) algorithm to design linear and nonlinear RF and microwave circuits," IEEE Trans. Microw. Theory Tech., vol. 53, no. 3, pp. 960-968, Mar. 2005.

[17] L. Zhang, J. Xu, M. C. E. Yagoub, R. Ding, and Q.-J. Zhang, "Efficient analytical formulation and sensitivity analysis of neuro-space mapping for nonlinear microwave device modeling," IEEE Trans. Microw. Theory Tech., vol. 53, no. 9, pp. 2752-2767, Sep. 2005.

[18] M. A. Ismail, D. Smith, A. Panariello, Y. Wang, and M. Yu, "EMbased design of large-scale dielectric-resonator filters and multiplexers by space mapping," IEEE Trans. Microw. Theory Tech., vol. 52, no. 1, pp. 386-392, Jan. 2004.

[19] K.-L. Wu, Y.-J. Zhao, J. Wang, and M. K. K. Cheng, "An effective dynamic coarse model for optimization design of LTCC RF circuits with aggressive space mapping," IEEE Trans. Microw. Theory Tech., vol. 52, no. 1, pp. 393-402, Jan. 2004.

[20] A. R. Conn, N. I. M. Gould, and P. L. Toint, Trust Region Methods, ser. MPS-SIAM Series on Optimization, 2000.

[21] M. H. Bakr, J. W. Bandler, K. Madsen, and J. Søndergaard, "An introduction to the space mapping technique," Opt. Eng., vol. 2, pp. 369-384, Dec. 2001.

[22] Q. S. Cheng, "Advances in space mapping technology exploiting implicit space mapping and output space mapping," Ph.D. dissertation, McMaster University, Hamilton, ON, Canada, 2004.

[23] em. ver. 9.52, Sonnet Software Inc., North Syracuse, NY.

[24] M. H. Bakr, J. W. Bandler, N. Georgieva, and K. Madsen, "A hybrid aggressive space mapping algorithm for EM optimization," IEEE Trans. Microw. Theory Tech., vol. 47, no. 12, pp. 2440-2449, Dec. 1999.

[25] MEFiSTo-3D Pro, version 4.0. Faustus Scientific Corporation, Victoria, BC, Canada, 2004.

[26] Empipe and Empipe3D. Agilent Technologies, Santa Rosa, CA, 1997.

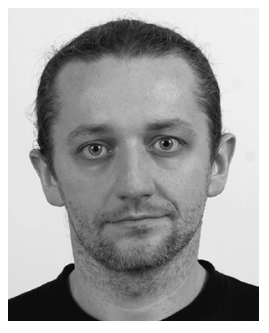

Slawomir Koziel (M'03) was born in Poland, in 1970. He received the M.Sc. and Ph.D. (with honors) degrees in electronic engineering from Gdansk University of Technology, Gdansk, Poland, in 1995 and 2000, respectively, and the M.Sc. degree in theoretical physics and mathematics and Ph.D. degree in mathematics (with honors) from the University of Gdansk, Gdansk, Poland, in 2000, 2002, and 2003, respectively.

He is currently a Post-Doctoral Fellow with the Simulation Optimization Systems Research Laboratory, Department of Electrical and Computer Engineering, McMaster University, Hamilton, ON, Canada. He has published more than 80 papers. His research interests include space-mapping-based modeling and optimization, circuit theory, analog signal processing, active filter design, evolutionary computation, and numerical analysis. 


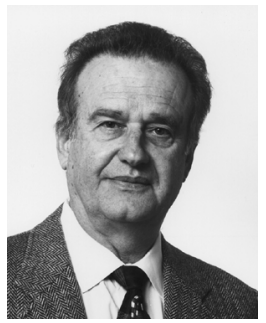

John W. Bandler (S'66-M'66-SM'74-F'78) was born in Jerusalem on November 9, 1941. He received the B.Sc.(Eng.), Ph.D., and D.Sc.(Eng.) degrees from the University of London, London, U.K., in 1963, 1967, and 1976, respectively.

He joined Mullard Research Laboratories, Redhill, Surrey, U.K., in 1966. From 1967 to 1969, he was a Postdoctorate Fellow and Sessional Lecturer at the University of Manitoba, Winnipeg, Canada. He joined McMaster University, Hamilton, ON, Canada, in 1969. He was Chairman of the Department of Electrical Engineering and Dean of the Faculty of Engineering. He is currently Professor Emeritus in Electrical and Computer Engineering, directing research in the Simulation Optimization Systems Research Laboratory. He has authored or coauthored more than 385 papers. He was a member of the Micronet Network of Centres of Excellence. He was President of Optimization Systems Associates Inc. (OSA), which he founded in 1983, until November 20, 1997, the date of acquisition of OSA by Hewlett-Packard Co. OSA implemented a first-generation yield-driven microwave computer-aided design (CAD) capability for Raytheon in 1985, followed by further innovations in linear and nonlinear microwave CAD technology for the Raytheon/Texas Instruments Joint Venture MIMIC Program. OSA introduced the CAE systems RoMPE in 1988, HarPE in 1989, OSA90 and OSA90/hope in 1991, Empipe in 1992, and Empipe3D and EmpipeExpress in 1996. OSA created the product empath in 1996 which was marketed by Sonnet Software, Inc. He is President of Bandler Corporation, which he founded in 1997. He joined the Editorial Boards of the International Journal of Numerical Modelling in 1987, the International Journal of Microwave and Millimeterwave Computer-Aided Engineering in 1989, and Optimization and Engineering in 1998. He was a Guest Editor of the International Journal of Microwave and Millimeter-Wave Computer-Aided Engineering Special Issue on "Optimization-Oriented Microwave CAD" (1997). He was Guest Coeditor of the Optimization and Engineering Special Issue on "Surrogate Modelling and Space Mapping for Engineering Optimization" (2001).

Dr. Bandler is a Fellow of the Canadian Academy of Engineering, the Royal Society of Canada, the Institution of Electrical Engineers, and the Engineering Institute of Canada. He is a member of the Association of Professional Engineers of the Province of Ontario, Canada, and the MIT Electromagnetics Academy. He received the Automatic Radio Frequency Techniques Group Automated Measurements Career Award in 1994 and the IEEE MTT-S Microwave Application Award in 2004. He was an associate editor of the IEEE TRANSACTIONS ON MICROWAVE THEORY AND TECHNIQUES (1969-1974) and has continued serving as a member of the Editorial Board. He was guest editor of the IEEE TRANSACTIONS ON MicrowaVE THEORY AND TECHNIQUES Special Issue on "Computer-Oriented Microwave Practices" (1974) and on "Automated Circuit Design Using Electromagnetic Simulators" (1997) and guest coeditor of the Special Issue on "Process-Oriented Microwave CAD and Modeling" (1992) and on "Electromagnetics-Based Optimization of Microwave Components and Circuits" (2004). He was chair of the MTT-1 Technical Committee on Computer-Aided Design.

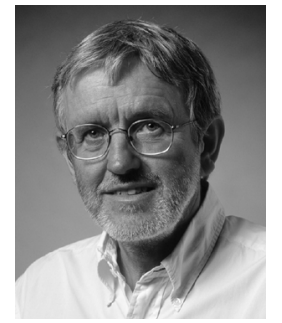

Kaj Madsen was born in Denmark in 1943. He received the cand.scient. degree in mathematics from the University of Aarhus, Aarhus, Denmark, in 1968 and the Dr.Techn. degree from the Technical University of Denmark (DTU), Lyngby, Denmark, in 1986.

From 1968 to 1988, he was a Lecturer in numerical analysis, apart from the 1973-1974 academic year, when he was with AERE Harwell, Didcot, U.K. Most of his career has been spent with the Department for Numerical Analysis, DTU. From 1981 to 1983, he was with the Computer Science Department, Copenhagen University, Copenhagen, Denmark. During summer 1978, he visited McMaster University, Hamilton, ON, Canada. In 1988, be became a full Professor. Since the 1990s he has arranged several international workshops on linear programming, parallel algorithms, and surrogate modeling, and space mapping. In 1993, he joined the Department of Mathematical Modelling, DTU, and during 1995-2000 was Head of that department. In 2000, he took an active part in forming the new department Informatics and Mathematical Modelling, DTU, which includes computer science and applied mathematics. Since January 2001, he has been Head of that new department. His primary fields of interest in teaching and research are nonlinear optimization, including space mapping techniques and global optimization, and validated computing using interval analysis. 